\title{
La soledad en los jóvenes de Neiva: comprensión semiótica de la desigualdad social ${ }^{1}$
}

\author{
Juan Camilo Puentes Castro \\ Magister en conflicto, territorio y cultura, Colombia \\ Juanpuentescastro@gmail.com \\ Claudia Marcela Pérez Bolívar \\ Magister en conflicto, territorio y cultura, Colombia \\ claudiamarcelaperezbolivar@gmail.co
}

\section{Resumen}

Con el paso de los años se han visto una serie de transformaciones en los diferentes centros urbanos del país (Almuna, et al. 2012; Baer, 2010). Una de las transformaciones más evidentes son las relaciones familiares en el hogar. Por ejemplo, en ciudades intermedias como Neiva, las relaciones han pasado de mantener elementos propios de la cultura rural a una cultura pseudo urbana con hogares en donde los padres están ausentes porque la prioridad no es la unión familiar sino la estabilidad económica. Las transformaciones traen consecuencias como la soledad de los hijos adolescentes, situación común que se encontró tanto en los jóvenes de estratos populares y altos. Lo anterior nos lleva a formular el siguiente interrogante: ¿De qué manera y qué implicaciones tienen las formas de asumir la soledad en los jóvenes de estrato social alto y del sector popular, entre 13 y 17 años, de la ciudad de Neiva?

La respuesta se construyó desde la sicología y la sociología, e interpretada desde la Semiótica (Sociosemiótica), por lo que el objetivo general de la investigación fue comprender la forma en la que se asumen la soledad en los grupos de jóvenes a investigar. Luego el marco interpretativo planteado se conocieron las implicaciones que tienen estas formas de asumir el fenómeno en la configuración de las condiciones de producción de sentido que terminan configurando socialmente lo real (Verón, 1987). Para esto se usó el enfoque cualitativo de recolección de información. Finalmente se concluyó que las condiciones estructurales son productoras de descapitalización social, para quienes no puedan adaptarse al nuevo sistema, que se manifiesta en la falta de confianza en sí mismos de parte de los jóvenes que no logran superar la condición de soledad, esto nos presenta como elemento principal la comprensión Semiótica de la desigualdad social debido a que se hace evidente el silenciamiento de aquellos que no pueden ganar la confianza suficiente para generar una voz propia, condenándolos a la sumisión social y la invisibilización.

Palabras Clave: Soledad, condición de juventud, Desarrollo psicosocial, Confianza en sí mismo, Sociosemiótica, condiciones de producción discursiva, desigualdad social.

1 El siguiente texto es la continuación de la investigación realizada por los autores para optar el título de magister en Conflicto, Territorio y Cultura, programa ofertado por la facultad de Ciencias Sociales y Humanas de la Universidad Surcolombiana y coordinado por el Dr. William Fernando Torres. El proceso de asesoría lo realizaron las profesoras Gema Trujillo y Milena Trujillo, su aporte al documento es infinito y por eso es necesario agradecerles por la constancia, la insistencia y los aportes (siempre constructivos). Luego fueron muchos quienes se sorprendieron por lo hecho y aportaron en demasía al manuscrito original, entre ellos Miguel Ángel Mahecha Bermúdez y Alejandro González Bastos a quienes les debemos la posibilidad de encontrar el otro con quien conversar, tan difícil en estos tiempos precarios y a quienes les debemos el componente semiótico que evidencia todo el desespero de lo que no comprendemos, pero la sapiencia del tiempo que obliga a esperar, respirar y pensar. 


\section{The loneliness in the young people of Neiva: semiotic understanding of social inequality}

\section{Abstract}

Over the years, a series of transformations have been seen in the different urban centers of the country (Almuna, et al., 2012; Baer, 2010). One of the most obvious transformations is family relationships at home. For example, in intermediate cities like Neiva, relationships have gone from maintaining elements of rural culture to a pseudo-urban culture with homes where parents are absent because the priority is not family unity but economic stability. The transformations bring consequences like the solitude of the adolescent children, a common situation that was found so much in the young people of high and popular strata. This leads us to ask the following question: In what way and what implications do the forms of assuming the loneliness in the young people of high social stratum and of the popular sector, between 13 and 17 years, of the city of Neiva?

The answer was built from psychology and sociology, and interpreted from the Semiotics (sociosemiotics), so the general objective of the research was to understand the way in which solitude is assumed in the groups of young people to investigate. Then, the interpretative framework was made, known the implications of these forms of assuming the phenomenon in the configuration of the conditions of production of meaning that end up socially configuring the real (Verón, 1987). For this the qualitative approach of data collection was used. Finally, it was concluded that the structural conditions are producers of social decapitalization, for those who cannot adapt to the new system, which is manifested in the lack of self-confidence on the part of young people who cannot overcome the condition of solitude, this presents us as a main element in the Semiotic understanding of social inequality because it becomes evident the silencing of those who cannot gain the confidence to generate a voice of their own, condemning them to social subjection and invisibilization.

Keywords: loneliness, condition of youth, psychosocial development, self-confidence, sociodemographic, conditions of discursive production, social inequality.

\section{De las grandes soledades, líbrame señor. Dame una soledad pequeña a la que pueda yo sobrevivirle Oración- Jader Rivera Monje}

\section{Planteamiento del problema}

Neiva es la capital del departamento del Huila y como tal se ha visto expuesta a múltiples transformaciones, recordemos que su posición geográfica la convierte en un punto álgido de la geografía colombiana en la medida que es referencia obligada para el tránsito hacia el sur del país, esas transformaciones han sido movidas siempre por fuertes oleadas migratorias, por ejemplo la ampliación de puestos públicos en los sesentas, el fortalecimiento de la extracción petrolera durante los setentas, la construcción de la hidroeléctrica de Betania, el cultivo de arroz a gran escala y la apertura de entidades bancarias del orden nacional en los años ochenta, sucesos que permitieron entender que en la ciudad circulaban capitales nunca antes vistos; este ha sido el recorrido realizado entre la "aldea grandota" que fuimos a la ciudad intermedia que somos.

La ampliación de su capacidad productiva, pero sobre todo de consumo ha generado cambios a nivel cultural, pues se dejaron abandonaron rasgos propios de la cultura rural como por ejemplo la frecuente presencia de los padres, la cercanía entre los hermanos, el compartir largos periodos de tiempo en el hogar, la cercanía con los vecinos, las formas de diversión, etcétera; 
por prácticas propias del capitalismo, en donde todos debemos correr detrás del dinero, esto lo evidencia Torres (2003) con el siguiente relato:

\begin{abstract}
“Los cultos religiosos y los guardias privados tal vez ayudan a paliar la zozobra cotidiana producida por las múltiples violencias. Los casinos quizás aportan dinero para salir de deudas o acceder a consumos aprestigiadores. Quizás, gimnasios, spas, diseños de sonrisas y escultura corporal ofertan las simetrías requeridas para fortalecer la autoestima $y$ ganar la capacidad de competencia que exigen estos tiempos. A la par, las zonas rosas siguen prometiendo espacios de diversión para tejer nuevas relaciones o escapar al agobio laboral [...] Todo esto sugiere que Neiva vive ahora entre la velocidad y la desesperación." (pág. 160)
\end{abstract}

Estas nuevas prácticas culturales se acentúan en la ciudad en esta nueva ola expansiva evidenciada por los altos índices de invención en el sector de construcciones, que se convirtió para el año 2013 en el principal renglón de inversiones en la ciudad (presentó un aumento del $282 \%$ frente al año inmediatamente anterior, siendo del 33\% su participación) seguido del sector comunicaciones (que aportó el $17.5 \%$ de inversiones) (Alcaldía de Neiva, 2010; Cámara y comercio, 2013).

Ahora, y siguiendo los mismos datos de apoyo, además de la evidencia empírica como ciudadanos, la expansión del casco urbano nos permite entre ver dos lógicas diferentes en las que se está creciendo: la primera que corresponde a la satisfacción de consumos, representada en centros comerciales y vivienda de alto valor; y la segunda lógica que corresponde a la respuesta al déficit de vivienda que hay para los sectores populares. Lo que hace evidente que la ciudad crece acentuando la brecha social.

La acentuación de estas demandas genera alteraciones en las dinámicas familiares, pues los responsables del hogar deben salir a laborar durante largas jornadas, lo que lleva a que los jóvenes pasen una gran parte del tiempo solos. Este tiempo en soledad se convierte en un problema cuando, apoyados por el informe epidemiológico de Neiva (Secretaria de salud de Neiva, 2013), se observa que los suicidios aumentaron de 8 casos (registrados) en el 2012 a 17 en 2013, también se puede ver en la prensa local que registró diversos casos de chicos y chicas durante el 2013, lo más sorprendente es que esta situación se hace evidente en ambos sectores sociales, aunque motivados por situaciones diferentes pero que terminan en esta nefasta forma de configurar simbólicamente las dificultades, el sin sentido y desesperación, en una ciudad que como diría Andrés Caicedo: "espera pero que no le abre las puertas a los desesperados" (2008)

Es por esto que la pregunta a la que responde esta investigación es: ¿De qué manera y qué implicaciones tienen las formas de asumir la soledad en los jóvenes de estrato social alto y del sector popular, entre 13 y 17 años, de la ciudad de Neiva?

\section{Marco teórico}

\section{Condición de juventud}

Para responder esta pregunta, y como condición de toda investigación que tenga como sujetos a los jóvenes, es necesario decir cómo se entiende y define al actor-objeto. Para este trabajo no se piensa lo joven como algo que se agota en lo biológico; por el contrario, se asume la categoría como algo movible y cambiante según el contexto en el que se ubique. Se entiende el desarrollo de los jóvenes anclado en el presente, por lo que se convierte en una etapa de la vida definitiva en la consolidación de la visión y el sentido que se le construye al mundo en pos de la autonomía y emancipación. Así pues, se comprende la juventud como una condición que se define como "el entrecruzamiento [...] de direccionamientos y posiciones que a modo de vectores de fuerza orienta, pero también localizan al sujeto, en un universo de oposiciones que ordenan el mundo social y los submundos, a modos de subcampos, en el que el sujeto actúa y deriva sus cualidades sociales" (Castellanos, 2010), es desde ahí donde surge para la juventud su Ser Social.

Sin embargo, la condición de juventud tiene elementos comunes a todos ellos y elementos 
que la matizan; en los elementos que emparenta y hace que la categoría tenga una existencia social se contempla lo que Castellanos (2010) llama la doble condición de miseria, que son la miseria de posición y la miseria de condición (Bourdieu y otros, 1999, p. 10). Pero estos elementos homogéneos son matizados por aquellos que los hacen dispares, por ejemplo la edad, el desarrollo biológico, la etnia, la generación, la moratoria psicosocial, moratoria vital, género, el acceso a las instituciones socializadoras, etcétera.

Pero de todos los elementos matizadores el más significativo es el socioeconómico (Reguillo, 2010), pues marca la forma en la que se accede al Capital Cognitivo u objetivo, entendido aquí como el mundo escolar como carta para superar la precariedad del estado actual; el Capital Social, entendido como la calidad de la relaciones sociales; y, el Capital Político, asumido como el reconocimiento y la toma de decisiones. Sin embargo, a pesar la importancia de los tres capitales que constituyen la experiencia humana es en el Capital Social donde todo converge, pues aquí se construyen los Referentes que permiten superar las carencias del individuo y es desde donde se genera la idea de desigualdad social como efecto propio de las condiciones objetivas o materiales.

\section{Soledad}

Es por eso que esta investigación toma como punto de partida el acceso al Capital Social y es la sensación de soledad la mejor forma de evaluar las diferentes relaciones que establecen los jóvenes pues este concepto se caracteriza por ser asumido como una experiencia subjetiva, involuntaria, negativa, atemorizante, a vergonzante; que afecta la esfera emocional, la esfera social, o ambas; además está basado en la calidad de las relaciones con los otros, no en el número; y que se relaciona con disminución en la autoestima, timidez, tristeza, desinterés por la vida.

Para entender la forma en la que se indagó por la soledad, acudiremos a Weiss, quien en palabras de Sánchez, 2009, diferenció entre soledad de tipo emocional y soledad de tipo social. "La soledad emocional aparece en ausencia de apego emocional, y ocurre como resultado de la pérdida de intimidad con personas cercanas como padres, pareja o hijos" en los jóvenes puede verse reflejada en la relación con los padres o la familia, puesto que de estas relaciones, propias del circulo primario de socialización, proviene el sentimiento de seguridad, evaluada por el apego y el sentimiento de guía; en contraste, "la soledad social aparece en ausencia de una red social de apoyo, por ejemplo, como consecuencia de la pérdida de amigos, vecinos y colegas" se puede ver reflejada en los jóvenes en las relaciones con los pares, puesto que estas producen la sensación de afiliación, compuesta por la integración social entendida como el compartir información y experiencias con los otros; $y$, el sentido de confianza mutua en donde se combate el sentimiento de vulnerabilidad y abandono.

\section{La soledad en el desarrollo psicosocial}

El preguntar por la forma en la que los jóvenes acceden al Capital Social y cómo la soledad los afecta, se convierte en algo relevante cuando nos apoyamos en el modelo teórico del desarrollo psicosocial de Erick Erickson. En este se divide el desarrollo de la vida humana en etapas, de estas la más importante es la que se ubica en el periodo de la pubertad y adolescencia, es decir el quinto estadío, pues se contempla un tránsito que va a marcar significativamente la vida del ser humano, este es el paso del circulo de socialización primario (Familia y padres) al círculo de socialización secundario (Pares) y de su superación dependerá la formación de la identidad personal, que se configura en aspectos como la identidad psicosexual, la identificación ideológica, la identidad psicosocial, la identidad profesional, la identidad cultural, religiosa; en últimas se configuran los significados de lo que somos y lo que nos define.

Según el mismo Erickson, para esta etapa, “el mayor problema con el que nos encontramos es el de quién pensamos que somos frente a quién piensan los demás que somos o que intentamos ser. ¿Quién cree él o ella que soy yo? Es una cuestión fastidiosa que uno se pregunta, y a la que es difícil encontrar una respuesta adecuada" 
(Erickson, 2000). La forma en la que se supera esta disquisición es a partir del desarrollo de $l a$ confianza en sí mismo que se tiene para pasar del primer círculo al segundo circulo de socialización; cuando la etapa se supera con dificultad se genera a largo plazo inseguridad e incertidumbre, es por eso que la Soledad se convierte en la crisis básica de la etapa de la juventud.

\section{La Semiótica Social}

La superación de esta crisis se convierte en un punto muy álgido en el bienestar de la juventud debido a que la Soledad es una zona de producción de sentido social relevante (2013), para comprender las dinámicas de estos actores sociales. Sin embargo, desde el marco interpretativo propuesto para esta investigación se quiere superar el mero estado de descripción y comprensión de la problemática planteada, propio de las ciencias sociales, por el contrario la semiótica social (Halliday, 1978) nos permite complementar esta mirada generando reflexiones frente a la forma en la que se construye la realidad social y sus implicaciones. Es entonces que asumiendo esta perspectiva el problema cobra otros matices, pues se convierte en el mejor hecho social para comprender el doble anclaje del sentido en lo social y lo social en el sentido (Verón, 1993). Esto quiere decir que entendemos la soledad y las formas en las que los jóvenes la asumen en primer lugar como un hecho social relevante para sus actores y por eso es necesario hacer evidente lo que se asume de esta (El sentido de lo social); pero en segundo lugar la extrapolamos mucho más allá y comprendemos que la soledad y la forma en la que se asume se convierte en productora del sentido (lo social en el sentido).

Esta distinción que retomamos de Verón es una propuesta que tiene como origen el

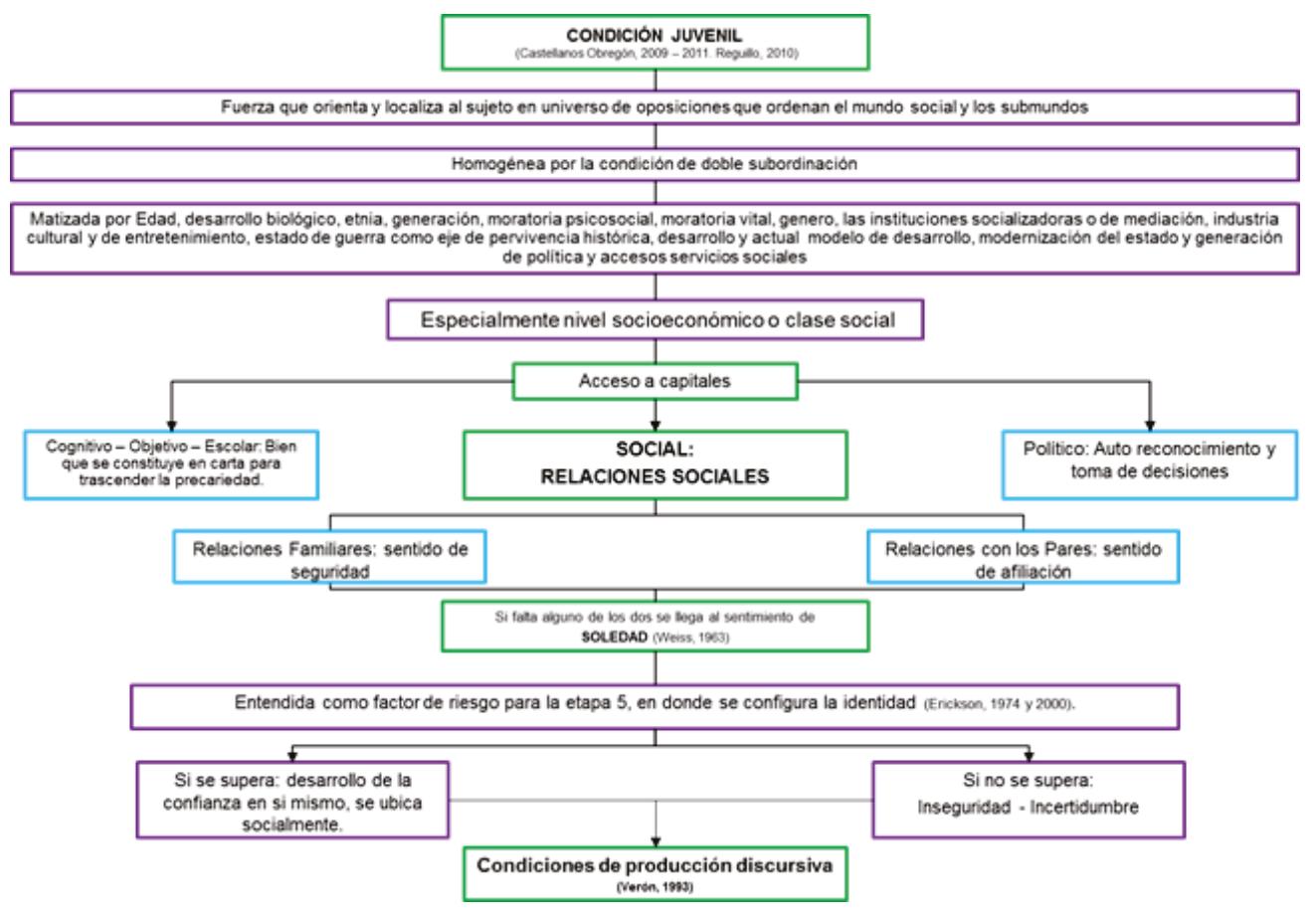

Gráfico 1. Articulación del marco teórico.

modelo ternario del signo lingüístico propuesto por Pierce, a diferencia del modelo binario (Saussureano) que contempla únicamente la relación Discurso-Objeto, este modelo se preocupa por el intérprete e interpretante, contemplando además las condiciones de producción y las condiciones de interpretación.
Para nosotros, asumir la soledad como un hecho social generador de sentido es entrar a describir las condiciones de producción discursiva en cada uno de los grupos de actores.

Es por esto que en esta investigación se parte del sentido de lo social y llega a la relevancia que 
tiene lo social en la construcción del sentido, es decir que el comprender cómo cada grupo de jóvenes asume la soledad nos permite además inferir como la manera de asumirla se convierte en condición de producción del discurso social y la forma como se construye la realidad social en cada nivel socioeconómico; y todo esto mediado por la Confianza en sí mismo, pues es la que genera la valoración y consolidación de la voz propia, y las implicaciones que acarrea el no superar la crisis básica.

\section{Metodología y Objetivos}

Esta investigación es de corte cualitativo, con un método de análisis comparativo entre las formas que asumen la soledad los jóvenes de los sectores populares y los del sector socioeconómico alto, la información se recogió por medio de la etnografía, revisión documental y la entrevista estructurada y semiestructurada. Disciplinariamente se apoya en la psicología y sociología con un marco interpretativo de origen semiótico, este diálogo disciplinar ocurre en la medida que el problema no surge del capricho investigativo, sino que surge luego de una descripción de contexto ampliar y rigurosa realizada durante el año 2013, que obliga al investigador a pensar la teoría en pro de la solución de un problema social relevante y no, como frecuentemente suele ocurrir en el mundo académico, construir los problemas en pro de hacer significativa una reflexión teórica.

Como objetivo general se planteó el analizar la manera en que se asume la soledad en los jóvenes entre 13 y 17 años del sector popular y del sector social alto en Neiva entre los años 2014 - 2015; en cuanto a los específicos, en la versión que se presenta para esta publicación, se busca en primer lugar realizar una descripción de los jóvenes del sector social alto y de los jóvenes del sector popular; en segundo lugar se pretende identificar la importancia de las relaciones familiares y con los pares en la soledad emocional y social de los jóvenes; en tercer lugar se Comparar la forma en la que asumen la soledad los dos grupos de jóvenes a comparar; finalmente se busca establecer las implicaciones que tienen estas consideraciones en la configuración de las condiciones de producción de sentido en los jóvenes.

\section{Resultados}

Inicialmente se realizó un acercamiento descriptivo a ambos grupos de jóvenes, los elementos que se contemplan en esta descripción son, entre otros: el sentido de sus nombres, información de sus padres (profesiones, tiempos, espacios en que conviven), la composición familiar, la idea que tienen de educación, la vida social, el uso que hacen del tiempo libre, la idea de futuro que tiene y finalmente la forma como definen la juventud. Luego se realizaron las entrevistas a profundidad, un dialogo extenso semiestructurado de corte biográfico analizado a partir de las categorías: Vivencias y consideraciones sobre la soledad, mundo escolar, relaciones familiares, relaciones con los pares, auto reconocimiento y toma de decisiones. Por grupo poblacional estos son algunos de los resultados obtenidos:

\section{Jóvenes del sector social Alto y la Soledad:}

Sus nombres recrean la tradición letrada de las familias, también hay asociaciones con nombres de grandes personajes de la política nacional y finalmente aquellos que recrean la tradición católica; los padres de estos chicos y chicas lograron acceder a estudios universitarios y de posgrado, las edades evidencian que sus hijos fueron concebidos a temprana edad y sostenidos por sus familias o padres que planearon para un momento de la vida más estable, asumiéndolos como parte esencial de sus proyectos de vida; los padres tienen muchas responsabilidades laborales, por lo que asume ciertos aspectos de la crianza en soledad o en compañías no tan relevantes (abuelas, nanas, empleadas del servicio doméstico, etc.).

Frente a sus ideas sobre la educación, este grupo de jóvenes asume la educación privada como un privilegio, aunque la asumen como una obligación, también se asume como forma de mantener el lugar que ocupan en la sociedad. Frente a la vida social, asisten a reuniones desde niños, esto los lleva a ganar independencia 
pensando en sus padres más como proveedores de bienes y servicios. En su tiempo libre tienen acceso a la televisión, servicios de paga por internet (Netflix, spotify, Apple Music), en el medio entienden que lo material les permite acceso a diversos productos, bienes y servicios; frente a la idea de sus futuros saben que deben estudiar, reconocen que su inserción al mundo laboral está dada por sus padres, pues estos son referentes de realización profesional pero no personal; finalmente la idea de joven que se tiene es construida por el tiempo que se tenga para experimentar (moratoria social).

Luego de haber realizado este proceso de descripción se puede afirmar que los jóvenes de este sector socioeconómico, son considerados como el principal proyecto de vida de sus padres, se perciben con multiplicidad de oportunidades, tienen como referente unos padres con logros profesionales pero que viven ausentes de sus vidas y que reemplazan la compañía por bienes, consumos, productos, oportunidades y servicios demás alto nivel, seenfrentan a grandes presiones sociales por mantener su nivel socioeconómico; además, estos consideran la educación como una forma de garantía para mantener el estatus que han tenido, pero al mismo tiempo lo consideran como una obligación; también podemos decir que viven día a día sin preocuparse por el futuro pues creen que sus padres se los garantizan, esa despreocupación por el futuro, sumado a la falta de autoridad de los padres les permite acceder a temprana edad a círculos en donde se consideran vitales los referentes mediáticos y en donde aparece el alcohol, las sustancias psicoactivas y las relaciones sexuales a temprana edad.

Frente al diálogo biográfico que se sostuvo se quiere dejar hablar con sus propias palabras:

"porque desde que me salí del Yumaná y pues desde antes siempre he tenido muchos choques con mi familia y muchas crisis existenciales porque como que nunca encajé en mi familia, como que nunca han aceptado mi pensamiento, mi forma de actuar, entonces siempre como que en esos momentos que me tocó pasar literalmente sola porque pues mi familia siempre me daba la espalda, pues a uno cuando está como en crisis y eso se le pasan cosas loquitas por la cabeza, pero yo por eso fue que me tatué Dios porque creo que él fue la persona que me sacó a mí de todas esas cosas negativas y de todos esas cosas malas que de verdad me estaban llenando en mi casa, porque yo siempre me la pasaba en mi cuarto llorando sola, o sea, sola y yo no sé, me empecé a pegar a Dios y le pedía mucha fuerza, que me ayudara a salir de eso y que me ayudara a entender todo lo que yo estaba pasando y por qué era y al final entendí que en el colegio pues con la ayuda de algunos profesores yo empecé, no sé, como a enfocarme, empecé a cambiar, me empezaron a gustar cosas que nunca antes llegué a pensar, en el Yumaná, que me gustaran, y es Dios le pone las batallas más fuertes a los guerreros más fuertes, y yo tengo más que claro que yo estoy hecha para cosas grandes." L.F.A.

"La calle me enseñó a enfrentar la soledad, desde pequeño, de pronto a veces tomaba mucho por eso, tomaba mucho, yo antes tomaba mucho, ahora no, la experiencia con Tato fue la más riata porque yo he sido un hombre muy fiel, cuando quiero, soy fiel de amigo, soy fiel de novio, así vainas así; a mi que me la claven y yo saber que no lo hice, o sea, eso me duele mucho, es la parte que yo creo que más me duele, y por eso me dolió tanto lo de este man porque yo era muy... o sea él me decía a tal lado y tal." C.M.A.

"Yo he aprendido a enfrentar la soledad a partir del hecho de estar aquí, y antes de eso yo creo que yo disfrutaba la soledad, porque yo no sabía que era estar sola, yo me sentía sola pero no sabía en serio qué era estar sola, antes de que todo esto pasara; yo creo que esta situación ha sido algo bueno, como algo que me ha ayudado a entender eso, como a valorar más las cosas. Es importante tener un propósito, el mío ahora, por ejemplo, es como graduarme, poder irme y ya." M.J.A.

\section{Jóvenes del sector social popular:}

En sus nombres se evidencia una fuerte influencia de la religión católica pues hace alusión a personajes bíblicos, otros están inspirados por reinas de belleza o personajes de la farándula de la época; los padres asumieron la paternidad a muy temprana edad insertándose en el mundo laboral para poder responder a sus nuevas 
condiciones, alcanzando como máximo nivel de estudios el bachillerato, sus hijos no son su principal proyecto de vida siendo tildados como "cargas" económicas; los hogares de sus padres suelen ser recompuestos, por lo que hay una pérdida paulatina de autoridad, las relaciones en estos nuevo hogares suelen ser tensas y conflictivas; también hay una marcada ausencia de los padres durante gran parte del tiempo, esta situación lleva a que los chicos y la chicas no tengan un interese en el sistema educativo, ni siquiera como obligación, razón por la que salir de la casa se convierte en algo primordial desde temprana edad.

Por los sectores donde viven son frecuentemente asociados a temas de delincuencia o sustancias psicoactivas razón por la que el tiempo libre se convierte en algo censurable por sus cuidadores, aunque ellos saben que no todos tienen esas prácticas y se pasa muy bien afuera de la casa, sus espacios son muy limitados quedándose una buena parte del tiempo en casa. Su definición de ser joven está relacionado con la estigmatización y que a pesar de mil dificultades aún tiene ganas de hacer muchas cosas, "descubrir el mundo", además ser joven está marcado por la falta de responsabilidad, la idea de diversión y el vivir el momento sin preocupaciones, razón por la que en sus tiempos libres juegan video juegos, ven películas, se conectan a internet, escuchan música o se van de "farra" (el tema de la fiesta en los jóvenes es un espacio a investigar)

Tienen una gran preocupación con la apariencia física, y entre ellos se clasifica en grupos o parches, es muy frecuente el uso del tatuaje como forma de expresión. Los espacios que ocupan en la ciudad suelen ser los de sus barrios, los colegios, algunos parques de la comunidad, es allí donde suelen estar con sus pares, cuando pueden. Es en este momento donde la música como lenguaje universal puede traducir algunas de las cosas que para estos chicos y chicas es difícil decir en palabras (esto fue frecuente durante la investigación, este grupo poblacional presenta unas fuertes dificultades a la hora de poder comunicar en ideas ampliamente articuladas sus propias experiencias) las traduce la música, de ahí su importancia en estos sectores, observemos lo que Porta (Rapero español) puede decir sobre lo que consideran una metáfora de su situación diaria: "Estoy solo en el silencio que molesta grito escucha mi última palabra, léete mi último escrito, préstame atención, solo pido eso, lo necesito. Caen gotas de sudor y de mis ojos en mi nota de suicidio" (canción: Nota de suicidio).

Por lo anterior y a modo de síntesis podemos señalar que la falta de referente de realización en la familia y su contexto no les permite creer en la posibilidad de acceder a otros contextos sociales y superar la visión resignada de la vida, que han ido construyendo ante la soledad y las dificultades económicas por las que han atravesado, así mismo la pérdida del rol de autoridad permite con mayor facilidad la generación de identidades con barras, pandillas, la música y la fiesta, las cuales se encuentran permeadas por altos consumos de alcohol, sustancias psicoactivas y una concepción de las sexualidad abierta.

Se afirma entonces que los jóvenes establecen diversas relaciones que están marcadas por la necesidad de generar una solución rápida a sus dificultades - familiares, sociales, económicas, afectivas- este inmediatismo les inhibe una concepción amplia de las posibilidades que tienen, desestimando la educación como forma de mejorar sus condiciones de vida y dificultando la construcción de un proyecto de vida a largo plazo.

Frente a esto, en sus propias palabras, se destacan estas afirmaciones:

"Mi papá, él estaba trabajando entonces mi papá llego a las tres la mañana a recogerme, porque cuando mi papá fue a la casa él encontró toda la ropa botada todo desordenada la casa entonces mi papa fue arriba la bienestar donde me cuidaban y él fue y me recogió." [...] "Mi papá es un amargado, a mi papá no le gusta nada, él le gusta mantener encerrado. Amargado llega de trabajar y se encierra, va a estudiar y se encierra; con mi papá no converso el solo es madrazo, todos los días llega bravo a echar a amargarme a mí, a echarme cosa en cara ahí." [...] "Nada. A mí me pueden decir, 
yo digo mientras que no se metan con mi papá se pueden meter con mi mamá, mientras que no se metan con mi pa vale "huevo". [...] "Mejor dicho yo siempre he contado con mi papá, él siempre me alcahuetea cuando está de buen humor siempre me alcahuetea las cosas cuando está de buen humor porque es un milagro que este de buen humor y él es un milagro un día que este buen humor que le regale a uno cosa, por lo menos así cuando esta amargado que dura cuatro o cinco días no le habla a uno, en el día no le habla uno" F.V.P.

"Me siento sola cuando extraño a mi tío o Natalia mi hermanastra, cuando peleo con mi mamá y eso sucede cuando no estoy de acuerdo con ella o con mi hermana porque yo soy una de las personas que no me gusta algo y empiezo y les digo, no me gusta que me compares y que obliguen a hacer cosas solo por capricho ellas, eso no me gusta...-, y pues eso".

"Pues antes cuando si peleaba con mi mamá me sentía mucho sola, y tendía a veces me cortaba, porque salía paleando con mi mamá, pero desde un tiempo ya deje de cortarme, ahora pienso en mi tío y lo recuerdo yo sé que a él no hubiera querido que me sienta sola no que me cortara o que nada de eso. Pienso que tengo que afrontar mis problemas no echarme para tras siempre para adelante".

"Un tío que murió a él lo quise mucho y me siento sola cuando me acuerdo de él con el yo viví toda mi niñez en Planada, sino que él era guerrillero y entonces él iba a salir de eso y pues yo a él lo quería mucho" D.G.P.

"A mí me dio muy duro la separación de mis papas porque mi preferencia siempre ha sido por mi papá y mi mamá mí no me dejaba hablar con él, me tenida distante de él, no me dejaba casi verlo entonces, me dolía mucho. Casi no comía, mantenía llorando. Y después ya empecé a verme con mi papá" [...] "Nunca me he sentido sola ni cuando mis papas se separaron, porque tenía mucho el apoyo de mis tíos, de mis abuelas, de mis hermanas." M.C.P.

\section{Asociaciones, deducciones e inferencias:}

Frente a la pregunta de investigación planteada se puede afirmar que los jóvenes del sector popular no presentan sensación de soledad, frente a los jóvenes del sector social alto que si manifiestan sentirse solos. Esta respuesta tiene elementos comunes, propios de la condición de juventud de ambos grupos sociales, en la medida que para los dos no es una preocupación la soledad social, pues el sentido de afiliación generado por la integración social se ve satisfecho por medios como la internet, la socialización en el colegio y en sus lugares de residencia, que les permiten encontrar pares con los cuales desarrollar actividades que tiene como fin producir satisfacción hedonista. En el desarrollo de la confianza mutua, se encontró que para ambos grupos esta recae en un solo par diferente del grupo amplio de pares; sin embargo, frente a este par los jóvenes del sector popular continúan teniendo dificultades para depositar toda su confianza, a diferencia de los jóvenes del sector social alto quienes suelen depositar la confianza en ese único par que en algunos casos asume también el papel de figura de apoyo y guía.

Frente a la soledad emocional, para ambos grupos, se evidencia que la figura de apego se ubica en el primer círculo de socialización, representado por la familia nuclear o extensa, estos son esenciales para el desarrollo de la seguridad, independientemente de si la relación es conflictiva o no. El otro elemento que es necesario para generar la sensación de seguridad es la figura de guía que para los jóvenes del sector popular se mantiene en el círculo primario, pues reconocen el sacrificio que han realizado sus padres para formarlos, razón por la que respetan las relaciones verticales que se dan con ellos, por lo que no se contraponen a los principios formados en su familia; sin embargo, esto hace que se genere una paradoja entre respetar los principios familiares y las satisfacciones de tipo hedonista propia de la etapa que están viviendo.

En contraste, para los jóvenes del sector social alto la figura de guía esta fuera del circulo primario, esto obedece a que las relaciones horizontales dentro de los núcleos familiares les permiten cuestionar los principios con los que han sido formados, lo que lleva afirmar que las relaciones con el segundo circulo de socialización ganan relevancia en la medida en 
que se convierten en referentes para replantear esos principios con los que fueron formados. Ante lo cual se puede afirmar que la soledad emocional se convierte en generador de sensación de soledad cuando la figura de guía no se encuentra en el círculo primario. En este contexto, este grupo afirma que sí se sienten solos, pues la soledad aparece cuando no hay nadie quien los escuche, ya sean los padres, los pares o las instituciones, por lo que asocian la soledad al reconocimiento de los jóvenes como actores sociales, pues cuando son escuchados saben que son importantes para alguien y desarrollan el sentido de confianza en sí mismos; es por eso que se puede decir que saben asumir la soledad de diferentes formas y que esta para ellos no es vista como un problema, sino como una situación que los va a hacer más fuertes, razón por la que deben enfrentarla, esto lleva a que sientan que tienen mejores recursos propios para poder afrontar con seguridad y autonomía los retos de la vida diaria.

En cuanto a los jóvenes del sector popular, se puede inferir que no experimentan soledad emocional, para los jóvenes de este sector también aparece el sentimiento de soledad cuando no hay quien los escuche o cuando no hay con quien hablar, sin embargo para este contexto no es posible que alguien los tome en cuenta pues las relaciones en sus familias y con las instituciones sociales son de subordinación, lo que hace que se acostumbren a ser silenciados. Esto conlleva a que los jóvenes se les dificulte el desarrollo de la confianza en sí mismos, que se ve reflejado en la forma cómo interactúan con el segundo círculo de socialización, en el cual la confianza mutua no hace parte de las relaciones que construyen con sus pares, evidenciando el temor que sienten frente a ser defraudados o rechazados, pues consideran que no cuentan con los suficientes recursos para afrontar los retos de la vida diaria.

\section{Discusión}

Lo anterior hace manifiesto que el grupo de jóvenes del sector social alto se sienten solos pero que esta sensación de soledad no se convierte en algo negativo en la medida que permite el auto reconocimiento y el desarrollo de la confianza en sí mismo, lo que configura un equipaje de fortaleza emocional-simbólica para superar diferentes dificultades que la vida trae; el grupo de jóvenes del sector popular por el contrario al no experimentar esta situación, sumado al papel de subordinación en el que son ubicados socialmente generan inseguridad e incertidumbre en sus vidas, en la medida que no cursan ese proceso de auto reconocimiento necesario y obligatorio para la formulación de una identidad personal.

Es desde este análisis que se evidencia en qué consiste la desigualdad social, superando la idea de que los problemas sociales están ligados al acceso a bienes, servicios y mercancías; ligándolos principalmente al capital social, es decir a los proceso de socialización (formas en que socializamos, tipos de socialización, características, etc.). Lo cual nos permite entender y evidenciar el lugar de origen de la desigualdad social en la ciudad y cómo el dialogo y la socialización son un punto fundamental en la configuración de perspectivas más amplias pues:

[Pensar] la "acción conjunta" es importante porque exhibe una especie de actividad sui generis en la cual las personas no actúan a partir de sus propios planes internos, guiones o cosas por el estilo, sino por estar entrelazados con las actividades de otros. En esta actividad, las acciones de las otras personas son una influencia formativa en lo que hacemos, tanto como cualquier cosa dentro nuestro. En tales circunstancias, el resultado general de tal actividad no depende de ninguno de los individuos involucrados. (Shotter, 1993)

Es decir, que limitar los diálogos, las complicidades, la construcción de relaciones sociales, etcétera, es generador de inseguridades pues noexiste una forma en la que seles reconozca la su voz frente a los demás; este problema, poco tangible $\mathrm{y}$ falto de reconocimiento, es reproductor de la miseria, una mucho más significativa que la que evidenciamos en la falta de bienes materiales, pues esta coarta la forma en la que se desarrollan los significados propios, la significación humana, es decir una propia manera de comprender los entramados 
del mundo en que vivimos, pues así como lo plantea Halliday "."[alguien] que aprende el leguaje aprende a "cómo significar"" es decir que cuando no se desarrolla la confianza en sí mismos y el auto reconocimiento desde el estar solos se inhibe la configuración del significar, el mismo Halliday plantea que esto es una necesidad básica (derecho fundamental):

Desarrollar un potencial semántico respecto de una serie de funciones del lenguaje que, en última instancia, son funciones sociales, representan modos de interacción entre el niño y los demás, así, el niño aprende a obrar lingüísticamente con carácter reciproco, y el lenguaje se transforma para él en un conducto primordial de socialización, porque esas funciones están definidas por los contextos sociales, en el sentido de Bernstein [...] Por tanto, la semántica del niño es funcionalmente especifica; lo que él desarrolla es una "semántica social", en el sentido de que constituye un potencial de significado vinculado a una serie particular de funciones sociales primarias (Halliday, 1982).

Y aquí el problema se convierte en algo mucho mayor, pues en este sentido entendemos cómo desde el uso cotidiano y ordinario del lenguaje se comunica un sistema social predeterminado, así es que desde las características de los procesos de socialización que tienen los jóvenes es como se configura la semántica básica de los individuos y junto a ella la línea base de las condiciones de producción del discurso que son los elementos configuradores de la forma cómo se enuncia el mundo, cómo se significa y se reordena según los propios criterios.

Lo que hacen los jóvenes que establecen una línea semántica básica que les permite establecer su propia configuración de sentido, independientemente de las condicione sociales a la hora de configurar el mundo, es que "ponen a prueba y verifican el discurso de los otros, lo cuestionan y desafían, lo reformulan u lo elaboran, etc. Porque en la práctica en el curso de una conversación, la comprensión compartida entre los participantes se desarrolla, se negocia o se "construye socialmente"'" (Shotter, 1993). Es por eso que en un contexto comunicativo cualquiera:
La "situación", que no es ni "mía" ni "suya", es algo a lo que ambos podemos contribuir: es "nuestra". Es una situación en la que "yo" siento que hice "mi" contribución, y en la cual "usted" puede sentir que hizo la suya. A menos que esto ocurra, yo podría sentir que estoy viviendo en su realidad o usted sentiría que está viviendo en la mía, o que ambos tenemos que vivir en una realidad que no es la nuestra. Es contra esto que se debe luchar en tales situaciones: si las realidades sociales se construyen socialmente, entonces es importante que todos podamos tener voz en el proceso de su construcción (Shotter, 1993).

Es entonces en este momento donde comprendemos como este problema de investigación se hace relevante en la medida que la desigualdad social está en la agenda pública, $\mathrm{y}$ frente a eso lo que este documento pretende es pensar desde otro punto de vista un problema social de índole estructural, asumiendo que el desarrollo de la voz propia, desde unas condiciones de producción de sentido que propicien el dialogo, el cuestionamiento y la reorganización, es un aporte significativo para la ciudad y los retos que acarrean los tiempos venideros.

\section{Conclusiones}

Podemos concluir que las maneras en las que se asume la soledad son configuradoras de la identidad personal de los jóvenes, el reconocerse solos lleva a que el grupo del sector social alto desarrolle confianza en sí mismos a partir del auto reconocimiento, generando una voz propia. Por el contrario los jóvenes del sector popular no se reconocen en soledad, por lo que se llenan de inseguridades e incertidumbres al no reconocerse, por lo que para ellos está reservado el silenciamiento al que han sido relegados históricamente.

Esto es relevante en la medida que son los elementos bases en la configuración de los contextos desde donde cada uno va a construir su forma de significar el mundo, este reconocimiento/silenciamiento es constructor de la semántica básica que configura las condiciones productivas de los discursos sociales. Es por 
esto que se genera la desigualdad social, pues lo que dicen unos es más importante (o tenido en cuenta) que lo que dicen otros, perpetuando una cultura de la desigualdad que condena a los sectores populares en la subordinación, el silenciamiento y el despojo histórico al que los hemos condenado.

A partir de aquí es necesario la construcción de retos urgentes frente a la forma en la que se generen procesos de socialización en los jóvenes. Reconocer que existen elementos no contemplados, y que esta investigación hace tangibles, que deben ser línea base para desarrollar trabajos educativo y de intervención social con los diversos grupos de jóvenes (de ambos sectores) desde sus propias condiciones contextuales.

Como ejemplo se puede decir que en primer lugar se debe repensar la necesidad que hay desde la política pública de juventudes en proponer espacios en donde dialoguen los diferentes grupos de jóvenes donde se puedan reconocer y auto reconocer; en segundo lugar, las escuelas deben entender que hay nuevos elementos para asumir las condiciones producción discursiva por lo que las necesidades y las metodologías en que se trabajan deben cambiar radicalmente, utilizando los contextos determinados de cada grupo para establecer sus prioridades, dado que así como los sectores populares deben desarrollar la voz propia, los sectores más favorecidos deben reflexionar sobre la necesidad de reconocer al otro; en tercer lugar, re pensar la política de medios de comunicación que propicie el reconocimiento de voces silenciadas; en cuarto lugar, se pueden construir actividades culturales que ayuden a resarcir las imposibilidades de la voz propia, entre otras que se conviertan en caldo de cultivo para poder valorar las formas como estos jóvenes silenciados pueden levantar sus voces de inconformismos, sin la necesidad de violentar al otro y reproducir la historia de violencias de nuestro país, pues es un reto como nación y como ciudad la necesidad del reconocimiento del otro como sujeto diferente, el reconocimiento de lo diverso como línea base de desarrollo social.

\section{Referencias bibliográficas}

Angulo. Y., \& Bonelo. I., \& Quesada, F., \& Zúñiga, F. (1994). Los imaginarios de los jóvenes estudiantes de secundaria de la ciudad de Neiva (tesis de especialización). Universidad Surcolombiana, Neiva, Colombia.

Alcaldía de Neiva-Secretaría local de Salud. (2014). Perfil epidemiológico municipio de Neiva- Huila del año 2013. Recuperado de http://www.alcaldianeiva.gov.co

Aliste Almuna, E., Contreras Alonso, M., \& Sandoval Manríquez, V. (2012). Industrialización, desarrollo y ciudad: transformaciones socio-demográficas $\mathrm{y}$ espaciales en la geografía social del gran Concepción (1950-2010). Revista INVI, 27(75), 21-71.

Baer, Luis. (2010). Transformaciones urbanas y de la estructura social de un barrio de la ciudad de Buenos Aires. Economía, sociedad $y$ territorio 10(33), 283-325. Recuperado en 26 de julio de 2017, de http://www. scielo.org. $\mathrm{mx} / \mathrm{scielo}$.php? script $=\mathrm{sci}_{-}$ arttext\&pid=S1405-84212010000200002\&ln $\mathrm{g}=\mathrm{es} \& \mathrm{tlng}=\mathrm{es}$.

Caicedo, Andrés. (2008). Mi cuerpo es una celdaAutobiografía. Bogotá: Editorial Norma.

Castellanos, J. M. (2011). La condición juvenil: opciones metodológicas para la construcción de un objeto de conocimiento. Jóvenes, culturas y poderes. Bogotá: Siglo del Hombre Editores.

Erikson, E. H., \& Miralles, A. G. (1980). Identidad: juventud y crisis. Barcelona: Editorial Paidós.

Halliday, M.A.K. (1994). El lenguaje como semiótica social. Bogotá: Fondo de Cultura Económica.

Monje, Jader. (2015). Antología. Bogotá: Arquitrave. 
Montero, M., \& Sánchez-Sosa, J. J. (2001). La soledad como fenómeno psicológico: un análisis conceptual. Salud mental 24(1), 1927 http://www.medigraphic.com/pdfs/ salmen/sam-2001/sam011d.pdf

Mejía, M. R. (2015). Las culturas juveniles: una forma de la cultura de la época. Educación y ciudad 18, 49-76. http://www.idep.edu. co/revistas/index.php/educacion-y ciudad/ article/view/168

Mahecha, M.A. (2011). Antología de lingüística cognitiva. Neiva: Editorial Universidad Surcolombiana.

Reguillo, R. (2010), “La condición juvenil en el México contemporáneo. Biografías, incertidumbre y lugares", en Reguillo, Rossana (Coord.) Los jóvenes en México. México: Fondo de Cultura Económica. Consejo Nacional para la Cultura y las Artes.

Shotter, Jhon (1993). El lenguaje en la construcción de sí mismo. Recuperado en 26 de julio de 2017, de https://es.scribd.com/ document/219255400/El-Lenguaje-y-laconstruccion-del-si-mismo-John-Shotter.

Torres, W. F., \& Lasso, L. E. (2003). In-sur-gentes: construir región desde abajo. Neiva: Editorial Universidad Surcolombiana.

Verón, E. (2013). La semiosis social, 2. Ideas, momentos, interpretantes. Buenos Aires: Editorial Paidós.

Verón, E. (1987). La semiosis social. Fragmentos de una teoría de la discursividad. Buenos Aires: Gedisa. 